University of South Florida

DIGITAL COMMONS

Digital Commons @ University of

@ UNIVERSITY OF SOUTH FLORIDA

South Florida

$11-1-2011$

\title{
Distress Tolerance Moderates the Relationship between Negative Affect Intensity with Borderline Personality Disorder Levels
}

\author{
Marina Bornovalova \\ University of South Florida, bornovalova@usf.edu \\ Alexis Matusiewicz \\ University of Maryland \\ Elizabeth Rojas \\ University of South Florida, elizabeth22@usf.edu
}

Follow this and additional works at: https://digitalcommons.usf.edu/psy_facpub

Part of the Psychology Commons

\section{Scholar Commons Citation}

Bornovalova, Marina; Matusiewicz, Alexis; and Rojas, Elizabeth, "Distress Tolerance Moderates the Relationship between Negative Affect Intensity with Borderline Personality Disorder Levels" (2011). Psychology Faculty Publications. 116.

https://digitalcommons.usf.edu/psy_facpub/116

This Article is brought to you for free and open access by the Psychology at Digital Commons @ University of South Florida. It has been accepted for inclusion in Psychology Faculty Publications by an authorized administrator of Digital Commons @ University of South Florida. For more information, please contact digitalcommons@usf.edu. 


\title{
Distress Tolerance Moderates the Relationship between Negative Affect Intensity with Borderline Personality Disorder Levels
}

\author{
Marina A. Bornovalova ${ }^{1}$, Alexis Matusiewicz ${ }^{2}$, and Elizabeth Rojas ${ }^{1}$ \\ ${ }^{1}$ Department of Psychology and Department of Mental Health Law and Policy, University of South \\ Florida \\ ${ }^{2}$ Center for Addictions, Personality, and Emotion Research, University of Maryland, College Park
}

\section{Abstract}

\begin{abstract}
A number of studies have suggested that negative emotionality and negative affect intensity play key roles in the development and maintenance of borderline personality disorder (BPD). However, more recent research indicates that one's response to affective discomfort may be an even more important variable in the pathogenesis of BPD than either negative emotionality or negative affect intensity per se. As such, the current study aimed to empirically test the moderating role of two well-validated laboratory measures of the ability to tolerate psychological distress (distress tolerance) in the relationship of negative emotionality and negative affect intensity with BPD levels a sample of 186 adult men and women drawn from the community and from an urban substance use center. Results provide laboratory-based evidence for a moderating effect of distress tolerance on the relationship of negative emotionality and negative affect intensity with levels of BPD. Specifically, the two former variables were related to levels of BPD among those with low distress tolerance. The current results add support to existing developmental frameworks of BPD, and suggest the importance of modifying one's response to affective distress along with levels of negative emotionality in treatment settings.
\end{abstract}

\section{Keywords}

Borderline Personality Disorder; Distress Tolerance; Affect Intensity; Negative Emotionality

\begin{abstract}
Borderline personality disorder (BPD) is a severe and persistent mental illness characterized by pervasive affective, cognitive, interpersonal and behavioral dysfunction, including emotional lability, interpersonal disturbances and engagement in risky or impulsive behaviors.[1-4] Epidemiological studies suggest that the rate of BPD in the general population ranges from $2-5 \%[5,6]$, although Trull [7] has estimated that as many as $13 \%$ of non-clinical adults demonstrate symptoms consistent with a diagnosis of BPD. Consistent with findings in clinical samples, subclinical levels of BPD are associated with significant
\end{abstract}

\footnotetext{
(C) 2010 Elsevier Inc. All rights reserved.

Correspondence: Address correspondence to Marina A. Bornovalova, Department of Psychology, University of South Florida, 4202 E. Fowler Ave, PCD4118G, Tampa, FL, 33620. Electronic mail may be sent to Bornovalova@usf.edu.

Publisher's Disclaimer: This is a PDF file of an unedited manuscript that has been accepted for publication. As a service to our customers we are providing this early version of the manuscript. The manuscript will undergo copyediting, typesetting, and review of the resulting proof before it is published in its final citable form. Please note that during the production process errors may be discovered which could affect the content, and all legal disclaimers that apply to the journal pertain.

No conflict of interest exists for any of the authors.
} 
social and occupational dysfunction as well as increased rates of mood, anxiety and substance use disorders [8].

Given the degree of distress and impairment associated with BPD, theoretical conjecture and empirical work have focused on the understanding of risk- and maintenance factors for the disorder. The dominant theory used to explain the pathogenesis of BPD is the diathesisstress model, which suggests that BPD results from the combination of biologically-based temperamental vulnerabilities and adverse and adverse childhood experiences such as childhood sexual, physical, or emotional abuse or neglect $[3,9,10]$. Consistent with this model, a growing body of research has focused on temperamentally-based emotional processes in BPD, including negative emotionality [4,11], affective instability [4], affect intensity [12,13], and emotion dysregulation [14].

The construct of negative emotionality (a heritable trait reflecting a tendency toward depression, anxiety, and poor reaction to stress) in particular has received a great deal of attention as a potential vulnerability for both the etiology and maintenance of BPD [4,11,1517]. Theoretical work suggests that negative emotionality underlies many of the risky and impulsive behaviors associated with BPD (i.e., deliberate self-harm, interpersonal aggression, substance use; [18-20]. Empirically, several cross-sectional studies report higher rates of negative affect among those with BPD compared to these without the disorder $[7,21,22]$. Moreover, a recent prospective study of children reported that negative emotionality in both early childhood and early adolescence prospectively predicted BPD features [15]. Finally, a study of adult psychiatric patients reported that BPD traits predicted both the recurrence and new incidence of major depressive disorder [2].

Another variable, negative affect intensity, has also been implicated as a major risk factor for BPD. On the surface, negative affect intensity might seem quite similar to negative emotionality. However, conceptual and empirical research makes an important distinction. Affect intensity refers to how strong an individual's negative affect is, regardless of the frequency or exact content of said negative affect. In other words, negative emotionality refers to the what (frequency and content of behavior), whereas affect intensity refers to the how (stylistic aspects of behavior of behavior) [23,24]. Follow-up empirical work also identifies negative emotionality and affect intensity as separate constructs. The correlation between affect intensity and negative emotionality is .3-.5 [23]; [25], and the correlation of the former with negative affect frequency is $\sim .2$ [25]. Affect intensity predicts symptoms of psychopathology above and beyond negative emotionality [26]. Finally, extraversion and neuroticism together account for $42 \%$ of the variance in negative affect intensity, which suggests that affect intensity captures elements of emotional experience not accounted for by these personality dimensions $[25,27]$. As such, negative affect intensity and negative emotionality can be thought of two correlated but distinct vulnerability factors.

The focus on negative affect intensity in BPD research is not surprising, as clinical lore describes individuals with BPD as "intense," especially in relation to negative affective states. Preliminary empirical evidence also reports moderate relationships with BPD pathology. For instance, Flett \& Hewitt [12] reported that high affect intensity was associated with self-reported BPD characteristics. Similarly, Levine et al [28] compared individuals with BPD to non-psychiatric controls. Results indicated that the BPD group showed significantly greater intensity of negative emotions than controls. In two clinical samples, affect intensity has been found to be especially elevated in individuals with BPD relative to those with bipolar II disorder [29], and was predictive of BPD features beyond depressive symptomatology [30]. 
Yet, there are reasons to believe that the association between negative emotionality and BPD is more complex than previously believed. First, it is worth noting that negative emotionality is normally distributed in the population [31], which suggests that, at least in the middle ranges of the distribution, it cannot be inherently pathological. Consistent with this conceptualization, Rosenthal, Cheavens, Lejuez, \& Lynch [32]reported that, although negative emotionality was a stronger predictor of BPD than childhood sexual abuse, thought suppression fully mediated the relationship between negative emotionality and BPD. Similarly, Gratz, Tull and Gunderson [33]found that the relationship between negative emotionality and BPD was partially mediated by experiential avoidance.

A parallel problem applies to the relationship between negative affect intensity and BPD. Several studies suggest that an individual's response to intense affective distress may be as important as affect intensity per se in predicting BPD traits. In a study examining physiological correlates of emotion among those with BPD, Herpertz et al., [13] examined affective responses to emotional stimuli using physiological and self-report measures. Results indicated that, while individuals with BPD report elevated subjective reactions to emotional stimuli, they did not demonstrate increased physiological reactivity relative to individuals without BPD [13,34]. These results may be interpreted as lower tolerance of emotional arousal rather than greater affect intensity among individuals with BPD[35]. Similarly, Cheavens et al., [36] and Gratz et al., [33] reported that the relationship between affect intensity and BPD symptoms was fully accounted for by (respectively) thought suppression and emotion dysregulation- that is, variables that encompass tolerance and acceptance of emotional distress in their definition [37]. Taken together, these findings suggest that it is not simply negative affect or negative affect intensity that is related to BPD traits, but how one responds to these vulnerability factors that may be critical in differentiating between those who develop BPD and those who do not.

The account of one's response to affective distress as a key variable in the development of BPD aligns this line of research with the larger literature focusing on the role of distress tolerance, or one's willingness and ability to persist in a positive behavior or to refrain from engagement in maladaptive behaviors during periods of physical or emotional distress $[3,38,39]$ in BPD. Distress tolerance figures prominently in theoretical conceptualizations of BPD (e.g. [3] and recent experimental findings have provided further evidence for diminished distress tolerance among individuals with BPD compared to healthy controls [40,41]. For instance, Bornovalova et al. [40] examined emotional distress tolerance in a sample of inpatient substance users with and without BPD. Results indicated diminished willingness to tolerate distress among participants with BPD. Poor emotional distress tolerance explained $18 \%$ of the variance in BPD status above and beyond demographic variables and Axis I psychopathology, suggesting the centrality of emotional distress tolerance to BPD. These studies are consistent with the notion of emotional distress tolerance as a moderator between negative emotionality and/or affect intensity and levels of BPD.

\section{Current Study}

The current study aimed to take the above line of thinking to its logical next step by empirically test the moderating role of distress tolerance in two established relationships: a) the association of negative emotionality with BPD traits; and b) the association between negative affect intensity and BPD. Notably, in assessing distress tolerance, we utilized two well-validated behavioral tasks (the Paced Auditory Serial Addition Task, [42]; and the Mirror Tracing Persistence Task, [43]. The use of the behavioral tasks for indexing distress tolerance allowed us to assess this construct without the contamination by distortions in responding that are so frequently associated with self-report instruments [44]. Finally, in 
order to obtain a truly representative dataset that includes the full distribution of BPD symptom levels (and is generalizable to both clinic and community samples), we sampled from an analogue sample of community adults and a sample of urban drug users seeking residential treatment for addiction.

\section{Participants}

Sample 1: Adult Community Smokers-Participants were 110 adult smokers enrolled in a larger study focused on understanding the predictors of long-term smoking cessation. Data from the initial/baseline testing session was used for the analyses in the current study. Participants were recruited through newspaper advertisements in the Washington, DC area targeted toward smokers with a desire to quit smoking and screened over the phone for inclusion/exclusion criteria. Inclusion criteria were all related to smoking behaviors and habits, in line with the goals of the larger study. Participants were excluded from this investigation if meeting any of the following: 1) presence of acute psychotic disorder, 2) use of psychotropic medications and/or nicotine replacement therapy (e.g., the patch); 3) the inability to give informed, voluntary, written consent to participate due to limited mental capacity. Participants from this sample were evenly split between genders (53\% male). The mean age (SD) was 46.9(10.05). Seventy percent of participants self identified as African American, $25 \%$, as Caucasian, $3 \%$ as other, and $1 \%$ as each Latino and Native American.

Sample 2: Inner-City Substance Users-Participants drawn from this sample were 76 inpatient residents in a drug and alcohol abuse treatment center in Northeast Washington DC. Typical treatment in the center ranges from 30 to 180 days. The center requires complete abstinence from drugs and alcohol (including any form of pharmacological treatment, such as methadone), with the exception of caffeine and nicotine; regular drug testing is provided and any substance use is grounds for dismissal. When needed, detoxification from an outside source is required prior to entry into the center. Aside from scheduled activities (e.g., group retreats, physician visits), residents are not permitted to leave the center grounds during treatment.

Participants were recruited into the study between 1-2 weeks of entering the Center, to minimize variability around withdrawal and long-term drug effects. The inclusion/exclusion criteria were similar to that in Sample 1. Participants were excluded from this investigation if they evidenced: 1) presence of acute psychotic disorder, 2) the inability to give informed, voluntary, written consent to participate due to limited mental capacity. Participants ranged in age from 18 to 62 , with a mean age of $42.21(S D=8.16)$. Sixty-seven percent $(n=51)$ of the participants were male. Eighty percent of participants self-identified as Black/AfricanAmerican, $11 \%$ as White, $1 \%$ as Hispanic/Latino, $1 \%$ as Native American, and $7 \%$ declined to report. With regard to highest education level achieved, $27.6 \%$ had not completed high school or received a GED, 32.9\% had completed high school or received a GED, 34.2\% had attended at least some college or technical school, and 5.2\% had completed college or beyond. The majority of participants were single (72.4\%) and unemployed (76.3\%).

\section{Measures}

Demographics Questionnaire-A short self-report questionnaire was administered to obtain information on age, gender, race, education level, marital status, and income.

Substance Use Disorders-The Structured Clinical Interview for DSM-IV Axis I Disorders (SCID; [45] was used to assess for the presence of drug and alcohol use disorders (which could potentially influence responses to the laboratory measures). Twenty-five percent of these interviews were reviewed by a PhD-level clinician (C.W.L.). In the three cases for which there was a discrepancy, a consensus was reached. With regard to the 
diagnoses in the sample, $20.4 \%$ had one substance use diagnosis; $13.2 \%$ had two; and 5.5\% had three. The rest did not meet criteria for any drug or alcohol abuse disorder. Drug use diagnoses (by drug choice) across samples are presented in Table 1 . The number of substance use disorder diagnoses was used as a potential covariate in the analyses below.

Personality Assessment Inventory-Borderline Features Scale (PAI-BOR; [46] - Levels of borderline personality disorder (BPD) were assessed by self-report using the PAI-BOR. The PAI-BOR consists of 24 items that are rated on a 4-point scale ranging from 1 (false) to 4 (very true).Studies on the psychometric properties of the PAI-BOR demonstrate that it has good internal consistency $(\alpha=.84)$, high test—retest reliability $(r=$. 86; [46], and good convergent and discriminant validity as demonstrated by strong relationships with diagnostic measures of BPD in both clinical [47] and community samples [7,47]. Internal consistency in the current study was good (Cronbach's $\alpha=.77$ )

In addition to providing an index of the overall frequency of BPD levels, the measure provides sub-scales for four symptom clusters: identity problems, affective instability, negative relationships, and self-harm. The identity problems sub-scale measures fears of abandonment and malleability of self-image, whereas affective instability measures sudden shifts in mood and intensity of emotion. The negative relationship sub-scale focuses on feelings of betrayal, loneliness, and instability in relationships, whereas the self-harm subscale focuses on impulsive and reckless behavior.

\section{Multidimensional Personality Questionnaire-Brief Form, Stress Reaction} Scale (MPQ-SR; [48]—The MPQ-BF is a 155-item version of the original 300-item MPQ [49]developed to assess a variety of personality traits and temperamental dispositions. Like the original MPQ, the MPQ-BF includes 11 primary trait scales which load onto three higher-order factors. The traits of Well-Being, Achievement, Social Closeness, and Social Potency load onto the higher-order factor of Positive Emotionality; the traits of Stress Reactivity, Alienation, and Aggression make up the higher-order factor of Negative Emotionality; the traits of Control, Harm Avoidance, and Traditionalism load on the higherorder factor of Constraint; and the trait of Absorption does not load on any of the higherorder factors. Scores from the traits scales of the MPQ-BF are highly correlated with the equivalent trait scales from the original MPQ ( $r$ 's ranged from .92 to .96) and have demonstrated high internal consistency (Cronbach's alphas range from 74 to .84; [48].

In the current sample, we utilized only the Stress Reaction scale as a "representative" index of negative emotionality. We chose this scale because it has the highest factor loading on the negative emotionality superfactor compared to the Alienation or Aggression scales (which load on the same superfactor). The relatively higher loading of MPQ-SR on negative emotionality has been shown in four different samples and across two MPQ formats (cite Patrick et al, 2002). As an additional benefit, the MPQ-SR is only 15 items long, making it easy to administer and curbing the risk of fatigue in our samples.

Negative Affect Intensity Measure (AIM, [23,50]-The AIM is a 12-item 6-point Likert-type scale ( $1=$ never, $6=$ always) developed by Bryant and colleagues [50] from the longer, full 40-item AIM measure (also see $[23,32,36]$ for psychometric evidence). The 12item AIM contains two subscales: AIM-Negative Intensity, tapping the chronic tendency to have intense experiences of negative emotions, and the AIM-Negative Reactivity, tapping into the tendency to become easily disturbed by emotional events [50]. Bryant et al., [50] indicated that the internal reliability was adequate for both subscales (Cronbach's $\alpha=0.70$ and 0.66, respectively). Because of the concern about the overlap of the Reactivity scale with Stress Reaction and Distress Tolerance, the current study used only the 6-item Intensity scale1. Internal consistency in the current study was good (Cronbach's $\alpha=.83$ ) 


\section{Behavioral measure of willingness to tolerate emotional distress 1: Paced Auditory Serial Addition Task (PASAT)-As our first behavioral measure of} willingness to tolerate emotional distress, we used a modified computerized version of the Paced Auditory Serial Addition Task (PASAT; [51]), which has been shown to produce elevated levels of emotional distress [42,43]. For this task, numbers were sequentially flashed on a computer screen, and participants were asked to add the presented number to the previously presented number before the subsequent number appeared on the screen. As the task was designed to limit the role of mathematical skill in persistence, the presented numbers only ranged only from 0 to 20 , with no sum greater than 20 . Participants were asked to provide answers by using the mouse to click on the correct answer on a number pad displayed on the screen, and were told that their score increased by one point with each correct answer and that incorrect answers or omissions would not affect their total score. The task consisted of four levels, the first three of which had varying latencies between number presentations. Level 1 (low difficulty) began with a 5-second latency, with each correct answer decreasing the latency by .5 second and each incorrect answer or non-answer increasing the latency by .5 second. The average latency across Level 1 was used as the latency for Level 2 (medium difficulty), and Levels 3 and 4 (high difficulty) utilized a latency that was half the value of the average latency from Level 1 . The first level lasted two minutes, the second level lasted two minutes, and the third level (which served as a prime for the final level) lasted one minute. Following a brief 2-minute rest period to complete the dysphoria ratings (see below), the final level began. The final level had the same latency between number presentations as the third level, but lasted five minutes and included an option to terminate the task at any time. Specifically, participants were informed that once the final level had begun they could terminate exposure to the task at any time by clicking a button on the computer screen labeled "Quit Task"; however the amount of money they would make at the end of the session would depend upon their performance on the task. Willingness to tolerate emotional distress was indexed as latency in seconds to task termination.

As a manipulation check to ensure that the task actually induced emotional distress, we examined pre- and post-task scores on a dysphoria scale, comprised of four items assessing levels of anxiety, frustration, irritability, and difficulty concentrating [42]. Each item was rated from 0 to 100, with the average pre- and post-task scores used for comparison. The post-assessment occurred following level 3 (in order to prevent differing durations in the final level from influencing the mood ratings). To provide a mild incentive for tolerating the emotional distress, participants were told that their performance on the task would influence their payment for the session.

\section{Behavioral measure of willingness to tolerate emotional distress 2: The Computerized Mirror-tracing Persistence Task (MTPT-C; [52,53])-In this task, participants were required to trace a red dot along the lines of a star using the computer mouse; however, the mouse was programmed to move the red dot in the reverse direction (i.e., if the participant moved the mouse to the left, the red dot would move to the right, etc.). To further increase the difficulty of this task (and its resultant frustration), moving the red dot outside of the lines of the star or stalling for more than two seconds caused a loud buzz to sound and the red dot to return to the starting position. Participants were told that although they could end the task at any time by pressing any key on the computer, their performance on the task would influence how much money they received. After receiving instructions, participants began the task and worked independently until they terminated the}

\footnotetext{
${ }^{1}$ However, we also reexamined the analyses using the total AIM (combination of the Reactivity and Intensity scales). The pattern of results was completely unchanged.
} 
task, or the maximum was reached. The participants were not informed of the maximum duration prior to beginning the task. Willingness to tolerate emotional distress was indexed as latency in seconds to task termination. Similar to the PASAT, pre- and post-task dysphoria scores were compared to provide a manipulation check; however, unlike the PASAT, only one level was used, and therefore the post-task rating could only be provided once the task was terminated or the 5-minute maximum duration was reached.

\section{Procedure}

All procedures were approved by the University of Maryland-College Park Institutional Review Board. The procedure was identical across the two samples. After providing written informed consent, eligible participants completed the self-report questionnaire packet including the measures described above. Participants were actively encouraged to seek assistance regarding questions that were unclear. While completing the questionnaires (which were ordered randomly across participants), participants were taken to complete the behavioral task at randomly selected times into a different room, and returned to complete their questionnaires once the task was completed. Participants were reimbursed $\$ 10$ to $\$ 20$ depending upon their performance on the tasks, with $\$ 20$ for persisting through both tasks, $\$ 15$ for persisting through only one task, and $\$ 10$ for terminating both tasks.

\section{Identification of Covariates}

Preliminary analyses were conducted to examine the effect of demographic factors (e.g., age, racial background and income) on the variables of interest, to identify potential covariates [54]. Gender, racial background, marital status, age, education, or income were not significantly associated with PAI-BOR score, nor were they associated with termination latency on the behavioral tasks. The number of substance use disorder diagnoses was related to PAI-BOR score $(r=.16, p<.05)$, but not to distress tolerance. Most surprisingly, sample membership was not related to either PAI-BOR or termination latency2. Because the multiple categories (frequently with few participants within each cell) could mask a true effect, we created dichotomous variables for race (African-American v. other) and marital status (single v. not), and a three-level variable for education (less than high school, high school graduate or GED, and college/technical school or above). We reexamined the relationship of these recoded variables with PAI-BOR; however, only education was related to PAI-BOR $[\mathrm{F}(3,181)=3.06 ; \mathrm{p}<.05)]$. Moreover, the effect for education approached significance for DT $[F(3,222)=2.54 ; p=.057)]$. Accordingly, education as well as number of substance use disorder diagnoses ere entered into the subsequent analyses as covariates.

Manipulation Check-As a manipulation check for the behavioral tasks, dysphoria scores were examined from pre-assessment to post-assessment for both tasks. Participants reported significant increases in dysphoria from baseline $(M=15.08, S D=18.86)$ to post-assessment following the PASAT $(M=28.79, S D=25.56, p<.001$, Cohen's $d=.61)$ and from baseline $(M=17.86, S D=19.59)$ to post-assessment on the MTPT $(M=29.91, S D=26.05, \mathrm{p}<.001$; Cohen's $d=.52$ ). Construct validity of the distress tolerance tasks was supported by a high correlation between the termination latencies of the PASAT and the MTPT $(r=.48, p<$. 001 ), and by comparable rates of premature termination on the PASAT (57.9\%) and the MTPT (66.8\%). As such, we created a composite score from the two behavioral measures as an index of distress tolerance by taking a mean z-score of the two duration latencies (referred from here as DT [distress tolerance]). Also of note, the number of errors made

\footnotetext{
${ }^{2}$ Because of the potential concern that we are sampling from very different populations, we re-examined the analyses with sample membership included as a main effect (despite it failing to reach significance at a univariate level). The pattern of results was very similar to what is reported in the manuscript, suggesting that the sample membership is having less of an effect than one would initially believe.
} 
during each task was not related to persistence on the respective tasks, nor was it related to PAI-BOR (all $p \mathrm{~s}>.35$ ), suggesting that the results are not attributable to executive functioning and/or skill.

Preliminary Univariate Analyses-Descriptive statistics and inter-correlations for the variables of interest are presented in Table 2. Notably, the correlations showed that AIM was strongly related to MPQ-SR, but was not related to either DT index. MPQ-SR was not related to either DT index. This indicates that AIM, MPQ-SR, and DT are separable constructs covering different content areas.

Next, we conducted a series of univariate linear regressions that controlled for number of substance use disorder diagnoses and education to assess the relationship of MPQ-SR, AIM, and DT with PAI-BOR. After controlling for the covariates, the relationship of MPQ-SR on PAI-BOR was significant $(\beta=.52, p<.001)$, and so was the relationship of AIM on PAIBOR $(\beta=.27, p<.001)$. The effect of DT on BPD scores was not significant $(\beta=-.10, p$ $=.20)$.

\section{Primary Analyses}

MPQ-SR, DT, and MPQ-SR by DT Interaction: A series of hierarchical linear regressions were fit in SPSS to examine the relationship of MPQ-SR and DT with BPD symptom severity. In the first block, we entered education, number of substance use disorder diagnoses, and the MPQ-SR score. Beta weights and changes in $R^{2}$ are presented in Table 3. The omnibus regression model was significant. The effect of substance use disorder diagnoses on PAI-BOR was marginally significant, and the effect of MPQ-SR was large and significant. In step 2, we entered the DT composite, which failed to reach significance and did not add significantly to the model. Finally, in the third step, we entered the two-way interaction between MPQ-SR and DT in relation to PAI-BOR. This effect was significant, and significantly improved the overall model fit $\left(R^{2}\right)$ as well. This final model indicates that MPQ-SR has a significant effect on PAI-BOR regardless of DT levels; however, the greatest PAI-BOR scores are observed among individuals with high MPQ-SR and low DT (see Figure 1 for a visual representation).

AIM, DT, and AIM by DT Interaction: To test the effects of AIM, DT, and their interactive effect on PAI-BOR, we again fit three hierarchical regression models. The notable difference is for these three models is, in these models, we controlled for MPQ-SR scores to assure that the interactions of interest are not redundant to the ones above. In other words, we wanted to make sure that the effect of AIM and the interaction of AIM with DT is not confounded by negative emotionality.

Beta weights and changes in $R^{2}$ for these models are presented in Table 4. In model 1, we entered education, number of substance use disorder diagnoses, the MPQ-SR score, and the AIM score. As expected, the omnibus regression model was significant. The effect of substance use disorder diagnoses on PAI-BOR scores was again only marginally significant; the effect of MPQ-SR was significant with a robust effect size; and the effect of AIM was not significant1. In step 2, we entered the DT composite, which also failed to reach significance, and the improvement in the overall model was negligible. However, when we entered the AIM by DT interaction in the last model (Model 3), the results evidenced a main effect of MPQ-SR, a main effect of DT, and a DT X AIM interaction (all ps <.05). The entry of the interaction term significantly contributed to improvement in model fit. This final model indicates that individuals with low DT are likely to display high PAI-BOR scores, and the greatest PAI-BOR scores are observed among individuals with high MPQ-SR and low DT (see Figure 2 for a visual representation). 
Post-hoc Exploratory Analyses-In all the above we used the PAI-BOR total score as the criterion variable. However, we believed that it would also be interesting to test how negative affect intensity, negative emotionality, and distress tolerance affect distinct PAIBOR symptom clusters: identity problems, affective instability, negative relationships, and self-harm. Thus, we replicated our analyses with each PAI-BOR subscale.

Our results indicated that all symptom clusters show the same pattern of relationships with AIM, DT, and MPQ-SR. Specifically, all four symptom clusters are strongly related to MPQ-SR, moderately to AIM, and not at all to DT. However, the interaction effects with DT are carried by the self-harm scale. That is, the MPQ-SR X DT and AIM X DT interaction are only found with the self-harm and total scales. This indicates that individuals with high negative emotionality and high affect intensity who are also low in distress tolerance are vulnerable to engaging in impulsive and reckless behavior.

\section{Discussion}

In the current study, we tested whether distress tolerance moderates the relationship of two emotional vulnerability factors, namely, negative emotionality and negative affect intensity, with BPD traits. Consistent with previous work, both vulnerability factors were related to BPD traits on a univariate level [16,30]. More importantly, however, was the evidence for the moderating role of distress tolerance.

Interestingly, the moderation "story" is slightly different for negative emotionality and negative affect intensity. In particular, the data suggests that negative emotionality places one at a risk for high levels of BPD, regardless of distress tolerance levels (although those with high levels of the former and low levels of later are most vulnerable). On the other hand, negative affect intensity is a risk factor for high BPD levels only in the context of low distress tolerance.

These differences notwithstanding, our results highlight the importance of an individual's response to affective distress as a risk factor for BPD. This aligns the current study with the broader conceptual and empirical work that discusses how fear and avoidance of internal sensations and emotions - more so than the actual affective state per se place one at risk for multiple types of psychopathology [40,55-57]. Although speculative, there is reason to believe that individuals who persist on distress tolerance behavioral tasks may do so by virtue of having higher levels of emotional acceptance and lower levels of thought and emotional suppression. An extension of the current work might include modeling the relationship between thought suppression and emotional avoidance in the context of the current model.

The current results are consistent with Linehan's [3] biosocial theory of the development of BPD - one of the most prominent theories of BPD etiology. This theory suggests that the inability to withstand emotional pain and attempts to terminate this pain at any cost underlies the range of maladaptive behaviors seen among BPD individuals (e.g., deliberate self-harm, suicide attempts, substance misuse). As such, the current study adds to the growing body of literature supporting the biosocial theory of BPD development.

Another interesting finding of the current study relates to the interrelationship between distress tolerance, negative emotionality, and affect intensity. In line with previous reports, we found that negative emotionality and affect intensity are highly correlated but distinct constructs, such that negative emotionality accounts for only $29 \%$ of the variation in affect intensity. Similarly, a recent review [58] brings up the question of whether distress tolerance is truly separable from negative emotionality. The small and non-significant correlation of 
distress tolerance with negative emotionality and affect intensity provides evidence that they are, indeed, separate constructs that are covering different behaviors.

Beyond theory, the current results have a number of clinical implications. In particular, the current data support the use of treatments that target difficulties with distress tolerance, such as Dialectical Behavior Therapy (DBT, [3] or Skills for Improving Distress Intolerance (SIDI, [38]. Indeed, if an unwillingness or inability to experience emotional distress results in the use of maladaptive coping strategies aimed at escaping or avoiding that distress among individuals with BPD traits, then improving distress tolerance may serve to reduce such maladaptive behaviors. Because the current study is cross-sectional, findings cannot speak to the developmental origins of BPD. However, should future research replicate the current moderator effect in longitudinal, developmental studies, treatment strategies drawn from DBT or SIDI might also prove effective in preventing the emergence of BPD.

Further, the current findings extend upon current empirical work on BPD by examining the extent to which emotional vulnerability factors identified in one clinical population can generalize to less traditional samples. Much of what is known about BPD is drawn from research conducted in a narrowly defined BPD sample, consisting mostly of Caucasian, middle-class female inpatients [59-61] while comparatively little is known about the phenomenology of BPD in clinically, ethnically and socioeconomically diverse samples. Although findings suggest that temperamental and environmental risk factors for BPD are largely consistent across clinical populations, there are also notable inconsistencies [5,14], highlighting the need to evaluate the fit of extant models to diverse samples before generalizing across clinical populations. By including both community-based smokers as well as inner-city substance users in residential treatment, the current sample reflects a broad spectrum of psychiatric severity, which, together, may be more representative of the actual phenomenology of BPD than samples recruited exclusively from inpatient samples or from the community.

While the present findings suggest that emotional vulnerability factors identified in earlier theoretical and empirical work do, in fact, generalize to less traditional samples, it is notable that there were some findings that were inconsistent with our hypotheses and with previous findings. Specifically, despite the prominent role of distress tolerance in some theories of BPD, we did not find that it was associated with BPD at a univariate level, which may reflect certain characteristics of the sample. For instance, given its association with many clinical disorders and problematic behaviors, distress tolerance actually not have a specific relationship to BPD, but may rather be associated with psychiatric severity more broadly. That is, low distress tolerance may be associated with BPD symptom severity only in the presence of other forms of psychopathology. Unfortunately, the sample size and methods of the current study were insufficient to test this notion, underscoring the need for future studies on this topic.

Several limitations of the current study should be acknowledged. First, the choice of the current sample raises questions about the generalizability of the results. However, by including community-based adult smokers in addition to substance dependent inpatients, the present sample is representative of a broad range of psychiatric severity and BPD symptomatology. Second, our sample size was rather modest; although this sample was sufficient to detect univariate and moderating relationships, it was insufficient to fully and accurately test other models. In particular, our sample size precluded two interesting analyses: whether the results vary by gender and whether they vary by drug of choice (nicotine versus cocaine versus heroin) [62-64]. Third, data from the current study are crosssectional in nature and in no way determine the role of either emotional intensity or distress tolerance in the development of BPD. Finally, although less of a limitation than a future 
direction, the current study used a dimensional conceptualization and measure of BPD traits. Given previous work indicating that self-report, dimensional scales and diagnostic measures show different patterns of reliability and validity [65], it would be interesting whether a diagnostic interview would yield the same pattern of results.

In conclusion, the current study provides preliminary support for the moderating role of distress tolerance in the relationship between negative affect intensity and BPD levels in a clinically diverse sample. This study lays the groundwork for larger-scale longitudinal studies that prospectively follow children and adolescents reporting elevated affect intensity and examine if distress tolerance differentiates between who develop BPD symptoms and those that do not by adulthood. Finally, findings from the current study have potential clinical implications, suggesting the utility of targeting one's response to affective distress, rather than affective distress per se.

\section{Acknowledgments}

Data for this project were collected at the University of Maryland, College Park. This work was supported by the National Institute of Drug Abuse Grant R36 DA021820 and P30 DA028807. All authors had full access to all of the data in the study and take responsibility for the integrity of the data and the accuracy of the data analysis.

\section{References}

1. Association, AP. Diagnostic and Statistical Manual of Mental Disorders. 4th ed.. Washington, DC: American Psychiatric Association; 1994.

2. Gunderson, JG. Borderline personality disorder: A clinical guide. Washington DC: American Psychiatric Publishing; 2001.

3. Linehan, MM. Cognitive behavioral treatment of borderline personality disorder. New York: Guilford Press; 1993.

4. Skodol AE, et al. The borderline diagnosis I: Psychopathology,comorbidity, and personality structure. Biological Psychiatry. 2002; 51(12):936-950. [PubMed: 12062877]

5. Grant BF, et al. Prevalence, correlates, disability, and comorbidity of DSM-IV borderline personality disorder: Results from the Wave 2 National Epidemiologic Survey on Alcohol and Related Conditions. Journal of Clinical Psychiatry. 2008; 69:533-545. [PubMed: 18426259]

6. Swartz M, et al. Estimating the prevalence of borderline personality disorder in the community. Journal of Personality Disorders. 1990; 4:257-272.

7. Trull TJ. Borderline personality disorder features in nonclinical young adults: I. Identification and validation. Psychological Assessment. 1995; 7(1):33-41.

8. Trull TJ, et al. Borderline personality disorder features in non-clinical young adults 2 . Two-Year Outcome. Journal of Abnormal Psychology. 1997; 106(2):307-314. [PubMed: 9131850]

9. Zanarini MC, Frankenburg FR. Pathways to the development of borderline personality disorder. Journal of Personality Disorders. 1997; 11(1):93-104. [PubMed: 9113824]

10. Zanarini MC, et al. The subsyndromal phenomenology of borderline personality disorder: A 10Year The subsyndromal phenomenology of borderline personality disorder: A 10-Year. American Journal of Psychiatry. 2007; 164(6):929-935. [PubMed: 17541053]

11. Depue, RA.; Lenzenweger, MF. A neurobehavioral dimensional model of personality disorders. In: Livesley, WJ., editor. The handbook of personality disorders. New York: Guilford; 2001.

12. Flett GL, Hewitt PL. Criterion validity and psychometric properties of the Affect Intensity Measure in a psychiatric sample. Personality and Individual Differences. 1995; 19(4):585-591.

13. Herpertz SC, et al. Affective responsiveness in borderline personality disorder: A psychophysiological approach. American Journal of Psychiatry. 1999; 156:1550-1556. [PubMed: 10518165]

14. Bornovalova MA, et al. Temperamental and Environmental Risk Factors for Borderline Personality Disorder among Inner-City Substance Users in Residential Treatment. Journal of Personality Disorders. 2006; 20(3):218-231. [PubMed: 16776552] 
15. Carlson EA, Egeland B, Sroufe LA. A prospective investigation of the development of borderline personality symptoms. Development and Psychopathology. 2009; 21:1311-1334. [PubMed: 19825270]

16. Trull TJ. Structural relations between borderline personality disorder features and putative etiological correlates. Journal of Abnormal Psychology. 2001; 110(3):471-481. [PubMed: 11502090]

17. Trull TJ, et al. Borderline personality disorder and substance use disorders: A review and integration. Clinical Psychology Review. 2000; 20(2):235-253. [PubMed: 10721499]

18. Chapman AL, Specht MW, Cellucci T. Borderline personality disorder and deliberate self-harm: Does experiential avoidance play a role? Suicide and Life Threatening Behaviors. 2005; 35(4): 388-399.

19. Kruedelbach N, et al. Impulsivity, coping styles, and triggers for craving in substance abusers with borderline personality disorder. Journal of Research in Personality. 1993; 7:214-222.

20. Silbersweig D, et al. Failure of frontolimbic inhibitory function in the context of negative emotion in borderline personality disorder. American Journal of Psychiatry. 2007; 164:1832-1841. [PubMed: 18056238]

21. Ball SA, et al. Personality, temperament, and character dimensions and the DSM-IV personality disorders in substance abusers. Journal of Abnormal Psychology. 1997; 106(4):545-553. [PubMed: 9358685]

22. Dougherty DM, et al. Laboratory measures of aggression and impulsivity in women with borderline personality disorder. Psychiatry Research. 1999; 85(3):315-326. [PubMed: 10333383]

23. Larsen RJ, Diener E. Affect intensity as an individual difference characteristic: A review. Journal of Research in Personality. 1987; 21(1):1-39.

24. Thomas, A.; Chess, S. The behavioral study of temperament. In: J, S.; F, F.; A, G., editors. The biological bases of personality and behavior. Washington, D.C.: Hemisphere; 1985. p. 213-226.

25. Schimmack U, Diener E. Affect intensity: Separating intensity and frequency in repeatedly measured affect. Journal of Personality and Social Psychology. 1997; 73(6):1313-1329.

26. Vujanovic AA, et al. Affect intensity: association with anxious and fearful responding to bodily sensations. Journal of Anxiety Disorders. 2006; 20(2):192-206. [PubMed: 16464704]

27. McFatter RM. Emotional intensity: Some components and their relations to extraversion and neuroticism. Personality and Individual Differences. 1998; 24(6):747-758.

28. Levine D, Marziali E, Hood J. Emotion processing in borderline personality disorders. Journal of Nervous and Mental Diseases. 1997; 185(4):240-246.

29. Henry C, et al. Affective instability and impulsivity in borderline personality and bipolar II disorders: Similarities and differences. Journal of Psychiatric Research. 2001; 36(6):307-312. [PubMed: 11684137]

30. Yen S, Zlotnick C, Castello E. Affect regulation in women with borderline personality disorder traits. Journal of Nervous and Mental Disease. 2002; 190(10):693-969. [PubMed: 12409863]

31. Trull TJ. Dimensional models of personality disorder: Coverage and cutoffs. Journal of Personality Disorders. 2005; 19(3):262-282. [PubMed: 16175736]

32. Rosenthal MZ, et al. Thought suppression mediates the relationship between negative affect and borderline personality disorder symptoms. Behavior Research and Therapy. 2005; 43(9):11731185.

33. Gratz KL, et al. Factors associated with co-occurring borderline personality disorder among innercity substance users: The roles of childhood maltreatment, negative affect intensity/reactivity, and emotion dysregulation. Comprehensive Psychiatry. 2008; 49(6):603-615. [PubMed: 18970909]

34. Herpertz SC, et al. Emotional responses in patients with borderline as compared with avoidant personality disorder. Journal of Personality Disorders. 2000; 14:339-351. [PubMed: 11204341]

35. Chapman AL, Gratz KL, Brown MZ. Solving the puzzle of deliberate self-harm: The experiential avoidance model. Behaviour Research and Therapy. 2006; 44:371-394. [PubMed: 16446150]

36. Cheavens JS, et al. An analogue investigation of the relationships among perceived parental criticism, negative affect, and borderline personality disorder features: the role of thought suppression. Behavior Research and Therapy. 2005; 43(2):257-268. 
37. Gratz KL, Roemer L. Multidimensional assessment of emotion regulation and dysregulation: Development, factor structure, and initial validation of the Difficulties in Emotion Regulation Scale. Journal of Psychopathology and Behavioral Assessment. 2004; 26(1):41-54.

38. Bornovalova MA, Daughters SB. How does dialectical behavior therapy facilitate treatment retention among individuals with comorbid borderline personality disorder and substance use disorders? Clinical Psychology Review. 2007; 27(8):923-943. [PubMed: 17376574]

39. Daughters SB, et al. Distress tolerance as a predictor of early treatment dropout in a residential substance abuse treatment facility. Journal of Abnormal Psychology. 2005; 114:729-734. [PubMed: 16351393]

40. Bornovalova MA, et al. A multimodal assessment of the relationship between emotion dysregulation and borderline personality disorder among inner-city substance users in residential treatment. Journal of Psychiatric Research. 2008; 42(9):717-726. [PubMed: 17868698]

41. Gratz KL, et al. An experimental investigation of emotion dysregulation in borderline personality disorder. Journal of Abnormal Psychology. 2006; 115(4):850-855. [PubMed: 17100543]

42. Brown RC, et al. Distress tolerance and duration of past smoking cessation attempts. Journal of Abnormal Psychology. 2002; 111(1):180-185. [PubMed: 11866171]

43. Lejuez CW, Kahler CW, Brown RA. A modified computer version of the paced auditory serial addition task (PASAT) as a laboratory-based stressor. Journal of Behavior Therapy and Experimental Psychiatry. 2003; 26:290-293.

44. Salters-Pedneault K, Tull MT, Roemer L. The role of avoidance of emotional material in the anxiety disorder. Applied and Preventive Psychology. 2004; 11(2):95-114.

45. First, MB., et al. Structured clinical interview for DSM-IV Axis I Disorders, ed. v. Nonpatient edition (SCID-NP. New York: NY State Psychiatric Institute; 1996.

46. Morey, LC. Personality Assessment Inventory: Professional manual. Odessa, FL: Psychological Assessment Resources; 1991.

47. Bell-Pringle VJ, Pate JL, RBrown C. Assessment of borderline personality disorder using the MMPI-2 and the personality assessment inventory. Assessment. 1997; 4(2):131-140.

48. Patrick CJ, Curtin JJ, Tellegen A. Development and validation of a brief form of the multidimensional personality questionnaire. Psychological Assessment. 2002; 14(2):150-163. [PubMed: 12056077]

49. Tellegen, A. Unpublished manuscript. Minneapolis: University of Minnesota; 1982. Brief manual for the Multidimensional Personality Questionnaire.

50. Bryant FB, Yarnold PR, Grimm LG. Toward a measurement model of the affect intensity measure: A three-factor structure. Journal of Research in Personality. 1996; 30(2):223-247.

51. Gronwall DMA. Paced auditory serial addition task: A measure of recovery from concussion. Perceptual and Motor Skills. 1977; 44:367-373. [PubMed: 866038]

52. Quinn EP, Brandon TH, Copeland AL. Is task persistence related to smoking and substance abuse? The application of learned industriousess theory to addictive behaviors. Experimental and Clinical Psychopharmacology. 1996; 4(2):186-190.

53. Strong, DR., et al. The Computerized Mirror Tracing Task Version 1. 2003. Unpublished Manual

54. Tabachnick, BG.; Fidell, LS. Using multivariate statistics. NY: Harper Collins; 1996.

55. Cheavens JS, et al. An analogue investigation of the relationships among perceived parental criticism, negative affect, and borderline personality disorder features: the role of thought suppression. Behaviour Research and Therapy. 2005; 43(2):257-268. [PubMed: 15629754]

56. Gratz KL, et al. A laboratory-based study of the relationship between childhood abuse and experiential avoidance among inner-city substance users: The role of emotional nonacceptance. Behavior Therapy. 2007; 38(3):256-268. [PubMed: 17697851]

57. Rosenthal MZ, et al. Thought suppression mediates the relationship between negative affect and borderline personality disorder symptoms. Behaviour Research and Therapy. 2005; 43(9):11731185. [PubMed: 16005704]

58. Leyro TM, Zvolensky MJ, Bernstein A. Distress Tolerance and Psychopathological Symptoms and Disorders: A Review of the Empirical Literature Among Adults. Psychological Bulletin. 2010; 136(4):576-600. [PubMed: 20565169] 
59. Silk KR, SHLee EM, Lohr NE. Borderline personality disorder symptoms and severity of sexual abuse. American Journal of Psychiatry. 1995; 152:1059-1064. [PubMed: 7793443]

60. Zanarini MC, et al. Treatment histories of borderline inpatients. Comprehensive Psychiatry. 2001; 42:144-150. [PubMed: 11244151]

61. Zanarini MC, et al. Severity of reported childhood sexual abuse and its relationship to severity of borderline psychopathology and psychosocial impairment among borderline inpatients. Journal of Nervous and Mental Disease. 2002; 190(6):381-387. [PubMed: 12080208]

62. Abrantes AM, et al. The role of negative affect in risk for early lapse among low distress tolerance smokers. Addictive Behaviors. 2008; 33(11):1394-1401. [PubMed: 18684569]

63. Chen KW, et al. An examination of mental health comorbidities within a residential substance use treatment program.

64. Simons JS, Gaher RM. The Distress Tolerance Scale: Development and validation of a self-report measure. Motivation and Emotion. 2005; 29(2):83-102.

65. Hopwood CJ, et al. A comparison of interview and self-report methods for the assessment of borderline personality disorder criteria. Psychological Assessment. 2008; 20(1):81-85. [PubMed: 18315403] 


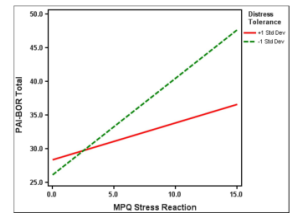

Figure 1.

Two-way interaction between DT and MPQ-SR on PAI-BOR 


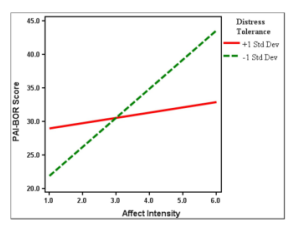

Figure 2.

Two-way interaction between DT and AIM on PAI-BOR 
Table 1

Drug Dependence Descriptive across Samples

\begin{tabular}{llll}
\hline \multicolumn{4}{c}{ \% Acknowledging Dependence } \\
\hline Drug Class & Both Samples & Smokers & Substance Users \\
\hline Alcohol & 14.5 & 1.9 & 39.2 \\
Marijuana & 7.2 & 3.2 & 15.2 \\
Crack/Cocaine & 28.5 & 4.5 & 75.9 \\
Opiates & 11.1 & 1.3 & 30.4 \\
PCP & 2.1 & 1.3 & 3.8 \\
Dependent on one drug & 20.4 & 6.4 & 48.1 \\
Dependent on two drugs & 13.2 & 0.0 & 39.2 \\
Dependent on three drugs & 5.5 & 1.9 & 12.7 \\
\hline
\end{tabular}




\section{Table 3}

The relationship of MPQ-SR, Distress Tolerance, Stress Reaction by Distress Tolerance Interaction to BPD traits.

\begin{tabular}{|c|c|c|c|c|c|}
\hline Predictor & Beta & $\begin{array}{l}\text { Full Model } \\
\quad \mathbf{R}^{2}\end{array}$ & $\Delta \mathbf{R}^{2}$ & $\Delta \mathbf{F}$ & \\
\hline \multicolumn{6}{|l|}{$\underline{\text { Model } 1}$} \\
\hline Education & -.11 & & & & \\
\hline Drugs & -.14 & & & & \\
\hline MPQ-SR & $.53^{* * *}$ & $.243^{* * *}$ & & & \\
\hline \multicolumn{6}{|l|}{ Model 2} \\
\hline Education & -.11 & & & & \\
\hline Drugs & -.13 & & & & \\
\hline MPQ-SR & $.52^{* * *}$ & & & & \\
\hline DT & -.04 & $.245^{* * *}$ & .002 & .39 & \\
\hline \multicolumn{6}{|l|}{$\underline{\text { Model } 3}$} \\
\hline Education & -.09 & & & & \\
\hline Drugs & -.14 & & & & \\
\hline MPQ-SR & $.51^{* * *}$ & & & & \\
\hline DT & .11 & & & & \\
\hline DT X MPQ-SR & $-.25^{* *}$ & $.279^{* * *}$ & .034 & $6.99^{* *}$ & \\
\hline \multicolumn{6}{|c|}{$\begin{array}{l}\text { Abbreviations: Drugs, number of substance use disorder diagnoses; MPQ-SR, Multidimensional Personality Questionnaire-Stress Reaction scale; } \\
\text { DT, Distress Tolerance. }\end{array}$} \\
\hline \multicolumn{6}{|l|}{ * $\mathrm{p}<.05$} \\
\hline \multicolumn{6}{|l|}{ *** ${ }^{*}<.01$} \\
\hline$* * * x<.001$ & & & & & \\
\hline
\end{tabular}


Table 4

The relationship of Affect Intensity, Distress Tolerance, and Affect Intensity by Distress Tolerance Interaction to BPD traits, Controlling for MPQ-SR.

\begin{tabular}{lcccc}
\hline Predictor & Beta & $\begin{array}{c}\text { Full Model } \\
\mathbf{R}^{2}\end{array}$ & $\Delta \mathbf{R}^{2}$ & $\Delta \mathbf{F}$ \\
\hline Model 1 & & & & \\
Education & -.11 & & & \\
Drugs & -.14 & & & \\
MPQ-SR & $.52^{* * *}$ & & & \\
AIM & .000 & $.243^{* * *}$ & & \\
Model 2 & & & & \\
Education & -.11 & & & \\
Drugs & -.15 & & & \\
MPQ-SR & $.52^{* * *}$ & & & \\
AIM & -.003 & & & \\
DT & -.045 & $.245^{* * *}$ & .002 & .39 \\
Model 3 & & & & \\
Education & -.11 & & & \\
Drugs & -.14 & & & \\
MPQ-SR & $.52^{* * *}$ & & & \\
AIM & -.02 & & & \\
DT & -.06 & & & \\
DT X AIM & $-.21 *$ & $.286^{* * *}$ & .041 & \\
\hline
\end{tabular}

Abbreviations: Drugs, number of substance use disorder diagnoses; MPQ-SR, Multidimensional Personality Questionnaire-Stress Reaction scale; AIM, Affect Intensity Measure; DT, Distress Tolerance.

*

p $<.05$,

*** $\mathrm{p}<.01$,

$* * *$

$\mathrm{p}<.001$ 